\title{
Implementation and Outcomes of a Maternal Smoking Cessation Program for a Multi-ethnic Cohort in California, USA, 2012-2019
}

\author{
Anne Berit Petersen ${ }^{1,6}$ (D) Temidayo Ogunrinu ${ }^{2} \cdot$ Shane Wallace ${ }^{2}$. Jane Yun ${ }^{3}$. Juan Carlos Belliard ${ }^{4,5}$. \\ Pramil N. Singh $\mathrm{h}^{2,4,6}$
}

Accepted: 15 October 2021 / Published online: 5 November 2021

(c) The Author(s), under exclusive licence to Springer Science+Business Media, LLC, part of Springer Nature 2021

\begin{abstract}
Smoking during pregnancy remains one of the most significant risk factors for poor birth outcomes. During 2012-2019, the Loma Linda University Health Comprehensive Tobacco Treatment Program (CTTP) used a multicomponent behavioral intervention for tobacco cessation for 1402 pregnant smokers with components of known efficacy (i.e., incentives, biomarker testing, feedback, and motivational interviewing). The CTTP cohort includes a multi-ethnic sample of pregnant women with a mean age of 27 years referred by collaborating community-based healthcare providers in San Bernardino county. Evaluation of program outcomes from 7 years of follow-up (2012-2019) creates a rich cohort dataset for implementation science research to examine the real-world effectiveness of the program. In this report, we provide a cohort profile, and 8-week prolonged abstinence (8-week PA) and relapse findings from the first year of follow-up $(n=233)$. We found: (1) $28.4 \%$ achieved 8-week PA, (2) At a median of 6.2 months of follow-up after achieving 8-week PA, $23.2 \%$ of enrolled subjects reported tobacco cessation, and (3) a high rate of loss to follow-up (44\%). In addition, our modeling indicated that the odds of relapse/smoking after enrollment was significantly higher in young mothers, non-Hispanic mothers (White, Black/AfricanAmerican), mothers in the first and third trimester, and rural mothers. Formative quantitative and qualitative research on the CTTP cohort will consider the effects of a range of implementation science (number of intervention sessions, addition of a mHealth component, distance to care) and individual (partner/household smoking, birth outcomes, NICU) outcome measures for the purpose of scaling up the CTTP model.
\end{abstract}

Keywords Smoking cessation $\cdot$ Pregnancy $\cdot$ Return to smoking/relapse $\cdot$ Program evaluation

Anne Berit Petersen

abpetersen@1lu.edu

1 Loma Linda University School of Nursing, 11262 Campus Street, Loma Linda, CA 92350, USA

2 Center for Health Research, Loma Linda University School of Public Health, 11234 Anderson St, Loma Linda, CA 92354, USA

3 Loma Linda University Health SACHS Clinic, 250 S G St, San Bernardino, CA 92410, USA

4 Loma Linda University School of Public Health, 11234 Anderson St, Loma Linda, CA 92354, USA

5 Institute for Community Partnerships, Loma Linda University, 11234 Anderson St, Loma Linda, CA 92354, USA

6 Transdisciplinary Tobacco Research Program, Loma Linda University Cancer Center, 11234 Anderson St, Loma Linda, CA 92354, USA

\section{Background}

Maternal smoking remains one of the most important modifiable risk factors for poor pregnancy outcomes in the U.S. and globally [1,2]. In addition to the risk of adverse health effects for the mother, tobacco use during the prenatal period is associated with poor fetal outcomes such as tobaccoinduced abortions and stillbirths, and numerous risks to the infant postpartum, including low birth weight (LBW), sudden infant death syndrome (SIDS), preterm birth, neurological and cognitive delays, congenital disabilities, colic, asthma and atopic pregnancies [1-3]. However, despite general awareness of these well-established risks associated with prenatal smoking, a significant number of U.S. women continue to smoke during pregnancy (20.8\% during 2007-2016) [1, 4].

Research has demonstrated that smoking cessation during pregnancy can reduce the risk of adverse health 
outcomes for infants, including decreasing the proportion of infants born with LBW [5] and prematurity [6, 7]. While a high proportion of women who smoke spontaneously quit before or in the early stages of pregnancy (up to $40 \%$ ) [8,9], the risk of postpartum relapse remains high (between 47 and 63\%) [10, 11]. Predictors of quitting during pregnancy and maintaining abstinence following childbirth include higher levels of education and socioeconomic status, being married or having a partner, Medicaid coverage or private insurance, partner/other household members do not smoke, planned breastfeeding, perceived adequate pre-natal care, and primiparity [12-14]. Behavioral factors include the lower levels of nicotine dependence, lower baseline cotinine levels, low exposure to secondhand smoking, no depression, and low stress during pregnancy [14]. Those who continue to smoke during pregnancy are more likely to belong to a disparity population by rurality, low income, or ethnicity, dealing with multiple stressors, or having higher nicotine dependence levels $[12,13]$. Moreover, the data also suggest that pregnant women underutilize existing smoking cessation services covered by Medicaid [15].

A recent systematic review (2019) found that health care providers are lacking in their provision of smoking cessation during pregnancy [16]. Further research is needed on the effectiveness of interventions and which interventions components are most effective in improving smoking cessation during pregnancy [16]. Thus, there continues to be a broad call for enhanced theory-based tailored smoking cessation programs for pregnant women, designed to effectively target identified barriers and facilitators of quitting and staying quit among this vulnerable population [13, 16, 17].
In San Bernardino County, Loma Linda University Health developed, through state funding (First 5 CA.gov), the Comprehensive Tobacco Treatment Program (CTTP) the county's most extensive maternal tobacco cessation program. During 2012-2019, CTTP utilized trained health educators to provide a multicomponent behavioral smoking cessation intervention for 1402 pregnant smokers using components with known efficacy (incentives, weekly testing, motivational interviewing) [5, 18]. The 7 years of follow-up (2021-2019) provides a rich source of data for evaluating implementation science outcomes [19] and mother-child outcomes in a multi-ethnic sample.

In this report, our objectives are to (1) profile the entire cohort $(N=1402)$, (2) describe implementation outcomes and individual-level variables that can be studied, and (3) provide an assessment of the real-world effectiveness of CTTP's first year (2012-2013). Real-world effectiveness of CTTP was assessed by 8-week, biochemically validated prolonged abstinence (8-week PA) and self-reported pointprevalence abstinence at 3-9 months post-intervention. Demographic and health variables associated with these outcomes are also examined.

\section{Methods}

\section{Cohort Profile}

During 7 years of follow-up, 1402 women were enrolled in CTTP (Fig. 1). Three of the 1402 women enrolled in the annual program also returned in a subsequent year (and pregnancy) for another attempt at smoking cessation. Annual

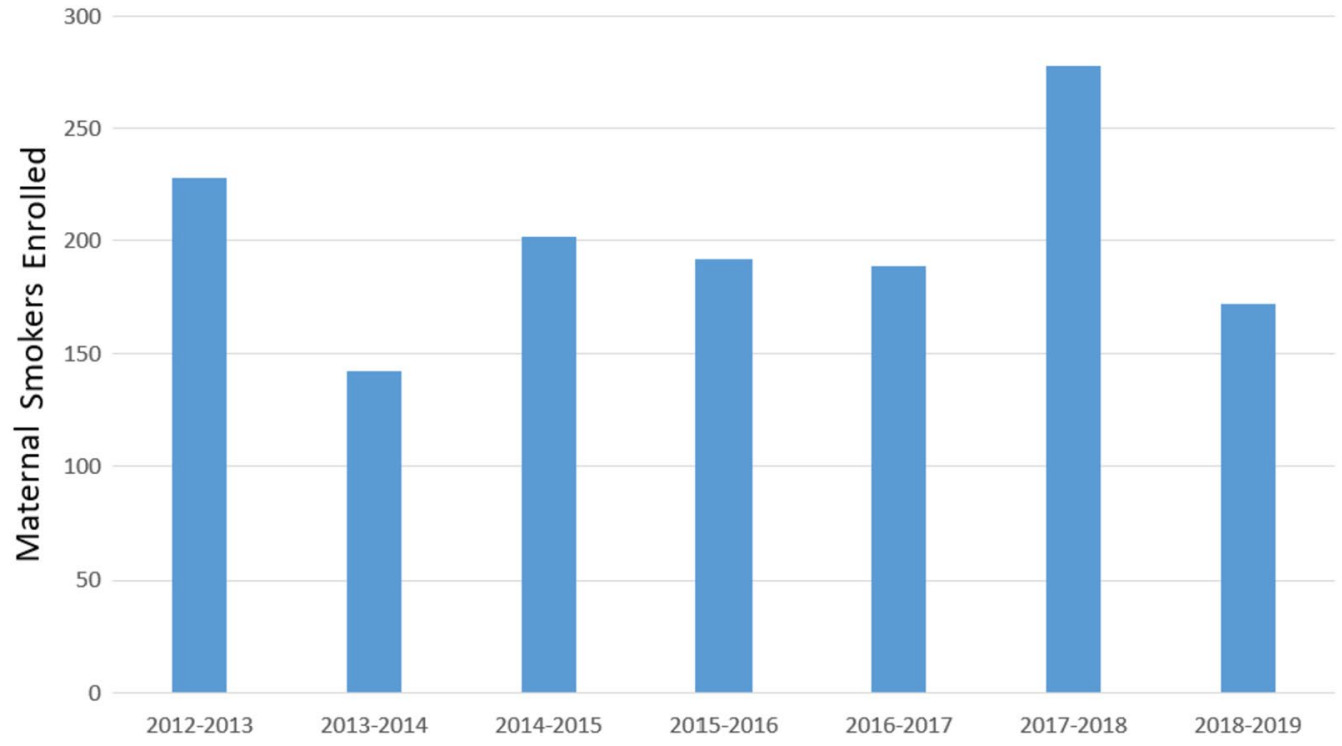

Fig. 1 Program enrollment by year for the Comprehensive Tobacco Treatment Program (CTTP) (2012-2019) $(N=1402)$ 
enrollment (not counting repeats) averaged 200 women per year. Individual level variables and implementation science outcomes collected during this follow-up are presented in Table 1. In this study, implementation science refers to the scientific study of methods to promote the uptake of research findings into routine care and improve healthcare 'services' effectiveness [20-22].

\section{Program Description}

\section{Coverage and Outreach}

The Comprehensive Tobacco Treatment Program model was designed to be an 8-week program, with weekly inperson meetings and telephone follow-up intervals at 3 , 6 , and 9 -months that were conducted by trained perinatal health educators (Bachelors degree or Masters in Public). Participants are referred to the program during prenatal visits by collaborating primary care providers at selected sites within San Bernardino County. The referral sites include outpatient clinics, hospitals, and rehabilitation homes from collaborating referral sites in four regional areas throughout the county. Patients are screened for tobacco use at the collaborating sites using current standardized prenatal protocols [23], and when indicated, referred to the CTTP for smoking cessation support (most often via fax). In addition, program announcements in the form of printed flyers are posted at WIC Offices (Special Supplemental Nutrition Program for Women, Infants, and Children) and distributed at community outreach events and health fairs with instructions on how to self-enroll. The flyers included the eligibility criteria (Are you pregnant \& smoking? Just quit smoking? Want to stay quit?) and contact information.

\section{Intervention}

The main goal of the CTTP is to support smoking cessation among pregnant women that are currently smoking or have smoked at any time during their pregnancy before the referral and point of enrollment. Upon receipt of the referral, perinatal health educators contact participants to schedule a face-to-face individual informal meeting at a mutually agreed-upon location or invite them to a small group session conducted at the premises of one of the referral sites. Most common meeting locations include public spaces near the participant's home, obstetric clinics, community partner agencies, hospitals, and outpatient rehabilitation centers. During the initial face-to-face visit, the perinatal health educators screened participants' current smoking behaviors by measuring urinary cotinine using NicAlert ${ }^{\circledR}$ test strips [24]. In addition, substance use and abuse screening (number of days a week during last month that participant used alcohol,

Table 1 Available mother-baby, household and implementation outcome variables in CTTP cohort data (2012-2019)

\begin{tabular}{ll}
\hline Individual outcomes & Implementation science outcomes
\end{tabular}

\section{Mother}

Date of Birth and age in years at enrollment

Estimated Date of Confinement (EDC)

Weeks gestation and trimester at enrollment

Gravida and Parity

Ethnicity

Smoking status at enrollment and weekly while enrolled in program

Number of cigarettes smoked per day at enrollment

8 weekly point-prevalence abstinence with negative urine cotinine

Smoking status at 2, 4, 6, and 9-month follow-up after EDC

Number of reported births

Baby

Birth outcomes (DOB, weeks gestation, weight, length, breastfeeding, NICU admission)

\section{Household}

Total family yearly income

Highest level of education

Total number of family members in home

Number of children under 6 and 18 years of age

Partner smoking status at enrollment

Number of other household members who smoke
Total number of intervention sessions attended

Number of inactive or dropped participants (only some years) by week

Referral method (How did you hear about CTTP?)

Repeat Enrollment, Repeated attempts with a single enrollment

Number date of successful follow-up calls at 3,6, and 9-months OR 2, 4, 6 (interval varied across program years) by region

Number and date of attempted follow-up calls at 3,6, and 9-months OR 2, 4, 6 (interval varied across program years) by region

Distance to program and referral sites

Referral site location vs. household location

Participation in mHealth text messaging sub-study (mother)

Text messaging language preference (English vs. Spanish)

Qualitative data (Key-informant interviews) exploring experience with the program, current smoking status, and quit attempts 
marijuana, and other drugs) and depression (10-item Edinburgh Postnatal Depression Scale) [25, 26] screening was conducted with referrals being made under the CTTP protocol as indicated.

The CTTP utilizes evidence-based materials developed by the American College of Obstetricians and Gynecologists, which recommended the 5 A's program $[23,27]$ and motivational interviewing techniques [5]. The health educator provides tobacco cessation-related education that highlights the risks associated with smoking during pregnancy and the benefits of cessation and works with the participant to develop an individualized quit plan. The purpose of the quit plan is to provide individualized support in setting a quit date and outlining personalized goals and strategies for quitting and staying quit. For those that had already quit, the plan focused on strategies for staying quit. At the weekly sessions, health educators review the individualized plan, support cessation efforts, and provide supplemental material based on the individual's needs. Weekly biomarker feedback using urinary cotinine is used to confirm abstinence. In addition, incentives (infant diapers and xylitol gum to prevent or reduce cravings) are provided each week that a participant tests negative for cotinine. Participants are considered to have completed the program when they have achieved prolonged abstinence, defined in this study as testing negative for urinary cotinine for eight consecutive weeks. However, it is important to note that a participant can be enrolled within the program for longer than 8-weeks, as they may not test negative for cotinine in the initial weeks of participation. After successfully completing eight (or more) weeks in the program, participants are followed up via telephone at 3,6, and 9-month intervals. During these follow-up telephone calls, participants are asked about the baby's well-being, their smoking status, and if they have relapsed, how much they are currently smoking (number of cigarettes/day), and when (date) they relapsed. If relapse has occurred, they are referred to the 1-800-NOBUTTS California Smoker's Helpline.

\section{Evaluation Methods}

We used a retrospective cohort design to evaluate the effectiveness of the CTTP during its first year in promoting smoking cessation among pregnant women and identifying predictors of prolonged abstinence (8-week PA). Human subjects' approval for the program evaluation was obtained from the Loma Linda University Institutional Review Board (IRB \# 5190418). The current analysis is based on a cohort of 233 pregnant women from San Bernardino County who were enrolled in the CTTP program during 1 year between July 2012 and July 2013.

8-week prolonged abstinence (8-week PA) (Outcome variable) defined by eight biochemically validated abstinence measures (urinary cotinine). Participants were determined to have completed the program if they stayed quit for eight consecutive weeks, as evidenced by negative urinary cotinine measured weekly. In addition, regardless of their completion status, during the first year, participants' smoking status (yes/no) was reassessed at 3,6, and 9 month intervals via telephone follow-up (Have you smoked during the past 7 days?).

\section{Covariates}

Gestational age was categorized into trimesters; women with gestational age $<13$ weeks were classified as the first trimester, those between 13 and 28 weeks as the second trimester, and those greater than 28 weeks as the third trimester. Smoking status at enrollment indicates whether or not the participant was still smoking at time of program enrollment as validated by biochemical feedback (urinary cotinine). The ethnicity/race variable had four levels: Asians, Blacks, Hispanics, and Whites. Age in years was obtained as a continuous variable.

\section{Statistical Analysis}

We used logistic regression to assess the effect of individual socio-demographic variables on smoking relapse. Relapse was defined as reporting smoking within any of the 8 weeks of program enrollment. All the analyses were conducted using SAS 9.3 [28].

\section{Results}

\section{Cohort Member Characteristics}

The CTTP cohort includes a multi-ethnic sample of women with a mean age of 27 years. The demographics for the entire cohort are presented in Table 2. During the first year, 233 enrolled in the CTTP, and they had a similar demographic profile to the entire cohort, as described in Table 2.

\section{Eight-Week Prolonged Abstinence (8-Week PA) Rate, Relapse, and Loss to Follow-Up}

During the first year of the program (2012-2013), CTTP enrolled 233 participants. At enrollment, $32.3 \%$ reported current smoking, while the remainder reported having recently quit. Figure 2 depicts the first-year participant's rates of 8-week PA, relapse, and loss to follow-up. We found that the immediate results of an 8-week intervention (post-quit date) indicated a 28.4\% (60/211) 8-week PA rate verified by weekly urinary cotinine tests. After a median of 6.2 months (range 3 to 9.8 months) of follow-up after 
Table 2 Demographic, behavioral, and gestational variables among enrollees in the Comprehensive Tobacco Treatment Program (San Bernardino County, California) during 2012-2019 $(N=1402)$

\begin{tabular}{ll}
\hline Mean age at enrollment (SD) & $26.79(5.75)$ \\
$\begin{array}{l}\text { Mean number of cigarettes per day (SD) at time of } \\
\text { quitting }\end{array}$ & $1.77(3.77)$ \\
Mean gestational weeks at delivery (SD) & $38.80(3.21)$ \\
Race/ethnicity & \\
Hispanic & $42.91 \%$ \\
Black/African-American & $19.60 \%$ \\
White & $30.08 \%$ \\
Native American & $0.78 \%$ \\
Pacific Islander & $1.57 \%$ \\
Others/multicultural & $4.92 \%$ \\
Trimester at enrollment & \\
First & $19.39 \%$ \\
Second & $33.00 \%$ \\
Third & $46.90 \%$ \\
\hline
\end{tabular}

achieving 8-week PA, $23.2 \%$ (49 reports of non-smoking out of 211 subjects) of all enrolled subjects continued to report not smoking. However, there was an overall $44 \%$ loss to follow-up among all subjects who enrolled in the program.

\section{Variables Associated with Relapse/Smoking During and After the Intervention}

We conducted a logistic regression analysis to identify variables associated with relapse/smoking during and after the intervention. In univariable models depicted in Table 3, we found that older mothers ( $\geq 31$ years) were threefold less likely to relapse relative to younger mothers (ages 21-25) (OR 0.33 95\% CI [0.15, 0.75]). Those mothers who had not adhered to a quit date at the start of the 8-week program (i.e., were current smokers) were three times more likely to relapse/smoke (OR 3.17 95\% CI $[1.44,6.95])$, and this effect was even more pronounced for the heaviest smokers (OR for $>5$ cigarettes per day $=4.68$ [1.36, 16.18]). Black (OR 2.84 95\% CI [1.07, 7.52]) and White (OR 3.93 95\% CI [1.75, 8.85]) mothers were more likely than Hispanic/Latino mothers to relapse/smoke. Also, the highest rates of relapse/smoking were found among participants in the first and third trimesters. Finally, when the remote rural regions of the county were compared to the urban area of the county, while not statistically significant, we observed a trend to higher rates of relapse during the 8 -week program among those in the rural regions (OR 1.64 95\% CI [0.73, 3.68]).

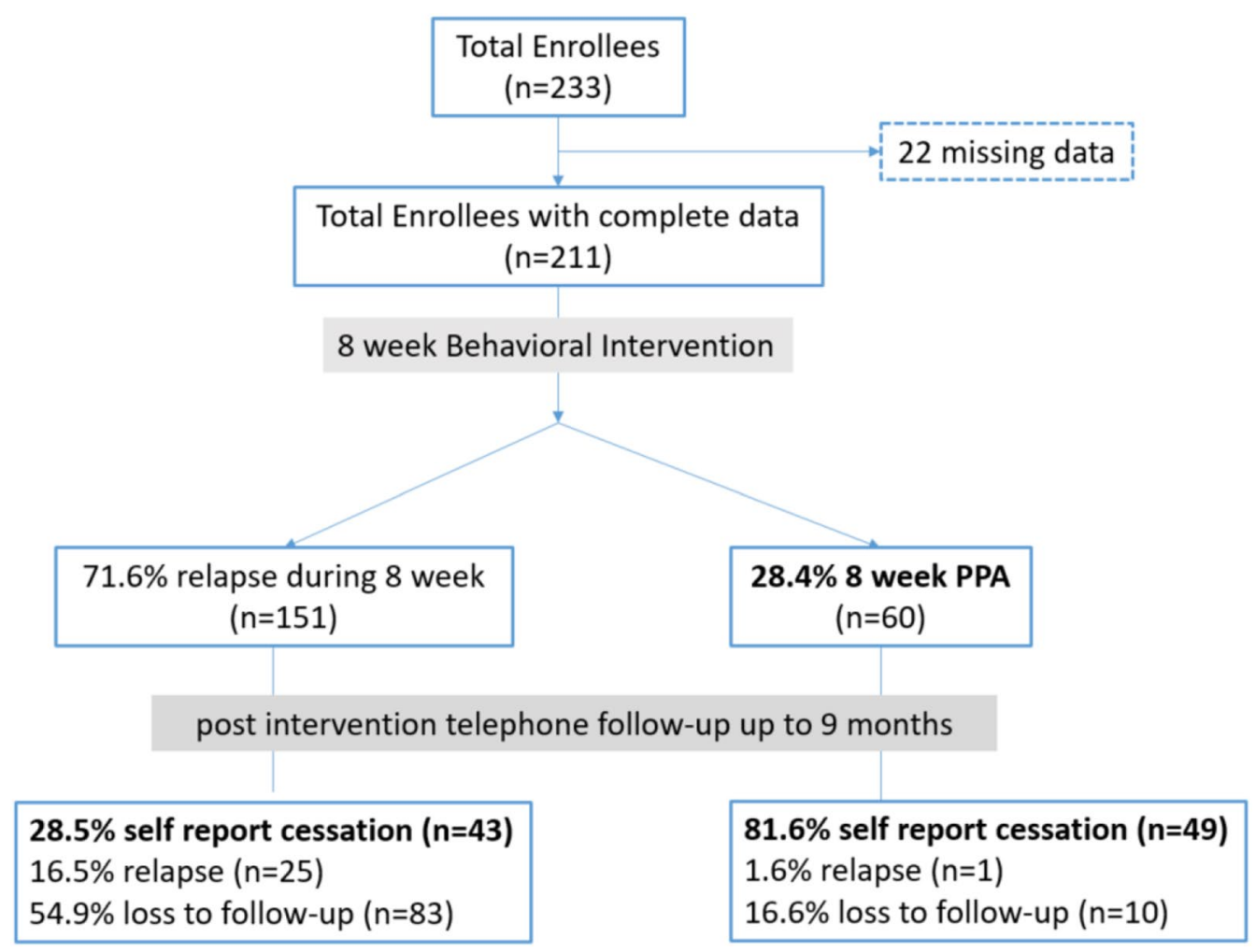

Fig. 2 Rates of biospecimen verified (urinary cotinine) 8-week prolonged abstinence (8-week PA), relapse, and loss to follow-up among the 233 enrollees in the first year (2012-2013) of the Comprehensive
Tobacco Treatment Program (CTTP) (San Bernardino County, California) $(n=233)$ 
Table 3 Univariable odds ratios relating selected variables to relapse during the 8-week program among first-year participants (20122013) enrolled in the Comprehensive Tobacco Treatment Program (San Bernardino County, California) $(n=233)$

\begin{tabular}{|c|c|c|c|}
\hline \multirow{2}{*}{$\begin{array}{l}\text { Covariates } \\
\text { Age }\end{array}$} & \multirow[t]{2}{*}{ OR } & \multicolumn{2}{|c|}{$\begin{array}{l}95 \% \text { Con- } \\
\text { fidence } \\
\text { Interval }\end{array}$} \\
\hline & & & \\
\hline 20 or younger vs. $21-25$ & 0.63 & 0.26 & 1.51 \\
\hline $26-30$ vs. $21-25$ & 0.59 & 0.26 & 1.33 \\
\hline 31 or older vs. $21-25$ & 0.33 & 0.15 & 0.75 \\
\hline \multicolumn{4}{|l|}{ Race } \\
\hline Asian/other vs. Hispanic & 1.31 & 0.42 & 4.12 \\
\hline NH Black vs. Hispanic & 2.84 & 1.07 & 7.52 \\
\hline NH White vs. Hispanic & 3.93 & 1.75 & 8.85 \\
\hline \multicolumn{4}{|l|}{ Education } \\
\hline 9th grade or less vs. college education or more & 1.64 & 0.72 & 3.70 \\
\hline $\begin{array}{l}\text { High school graduate vs. college education or } \\
\text { more }\end{array}$ & 1.45 & 0.66 & 3.16 \\
\hline \multicolumn{4}{|l|}{ Income } \\
\hline$\$ 10,000-\$ 25,000$ vs. more than $\$ 25,000$ & 1.05 & 0.37 & 2.98 \\
\hline Less than $\$ 10,000$ vs. more than $\$ 25,000$ & 1.77 & 0.67 & 4.67 \\
\hline \multicolumn{4}{|l|}{ Single parent } \\
\hline Yes vs. no & 1.49 & 0.76 & 2.92 \\
\hline \multicolumn{4}{|l|}{ Partner smokes } \\
\hline Yes vs. no & 1.59 & 0.86 & 2.94 \\
\hline \multicolumn{4}{|c|}{ Smoking status at time of enrollment (current smoking vs. quit) } \\
\hline Yes vs. no & 3.17 & 1.44 & 6.95 \\
\hline \multicolumn{4}{|l|}{ Cigarettes } \\
\hline 5 or less/day vs. non-smoker & 2.17 & 0.84 & 5.62 \\
\hline More than 5/day vs. non-smoker & 4.68 & 1.36 & 16.18 \\
\hline \multicolumn{4}{|l|}{ Alcohol use } \\
\hline Yes vs. no & 0.99 & 0.52 & 1.89 \\
\hline \multicolumn{4}{|l|}{ Drug use } \\
\hline Yes vs. no & 1.65 & 0.72 & 3.78 \\
\hline \multicolumn{4}{|l|}{ Gestational age at enrolment } \\
\hline First trimester vs. second trimester & 4.04 & 1.28 & 12.72 \\
\hline Third trimester vs. second trimester & 2.36 & 1.23 & 4.50 \\
\hline \multicolumn{4}{|l|}{ Parity } \\
\hline 2 vs. 1 & 0.74 & 0.31 & 1.76 \\
\hline 3 vs. 1 & 0.96 & 0.38 & 2.46 \\
\hline 4 vs. 1 & 0.91 & 0.35 & 2.41 \\
\hline 5 or more vs. 1 & 0.74 & 0.30 & 1.84 \\
\hline \multicolumn{4}{|l|}{ Number of children } \\
\hline 1 vs. none & 0.91 & 0.42 & 1.99 \\
\hline 2 vs. none & 1.04 & 0.43 & 2.53 \\
\hline 3 or more vs. none & 0.82 & 0.36 & 1.87 \\
\hline
\end{tabular}

\section{Discussion}

Our CTTP cohort profile and report of first-year findings focus on a maternal smoking cessation program in San
Bernardino County, CA-the largest county in the contiguous U.S.-in which nearly $12 \%$ of pregnant women reported having smoked during the 3 months before their pregnancy (vs. $10.8 \%$ statewide) $[29,30]$. The disparity trends we find in the first-year modeling of relapse in the CTTP cohort (i.e., higher in young mothers, non-Hispanic mothers (White, Black), mothers in the first and third trimester, and rural mothers) closely follow county, state, and national trends on the overall burden of maternal smoking. Specifically, county, state, and national trends indicate higher maternal smoking rates in rural mothers, mothers without private insurance, mothers with less than high school education, and non-Hispanic Black or White mothers [1, 4, 29-33].

Our approach to analyzing data from the entire cohort profiled here will be to consider these disparities for maternal smoking and relapse smoking under the socioecological model used to develop the domains of the National Institute on Minority Health and Health Disparities (NIMHD) Research Framework [34]. Under this framework, theorydriven analysis and optimization of the multicomponent CTTP program should include components that address multiple domains of the NIMHD Framework: behavioral, environmental, sociocultural, and healthcare systems [34]. Individual and implementation science outcome variables listed in Table 1 provide a rich data source for this process. Here, we discuss real-world effectiveness demonstrated in year 1 and the scope of future research on implementation science and individual outcomes for the entire cohort.

\section{Real-World Effectiveness of Year 1}

Our program outcome findings for 2012-2013 support the effectiveness of the 8-week incentivized health educatordelivered model. Specifically, we found a $28.4 \%$ 8-week PA rate after the intervention and that during a median of 6.2 months of follow-up after achieving 8-week PA, 23.2\% self-reported no current use of tobacco. Recent systematic reviews of individual behavioral interventions outcomes of the CTTP appear to be comparable to interventions provided to other multi-ethnic populations of pregnant women who smoke; however, further analysis of the entire cohort is needed in order to make meaningful comparisons [3, 5].

The cessation rates observed during year 1 of CTTP are similar to other multicomponent interventions in pregnant smokers [3, 5, 35]. However, when considering the realworld effectiveness of this program, it is of concern that the rate of loss to follow-up (44\%) is substantially higher than in other intervention studies [5, 36, 37]. In studies conducted among pregnant women, loss to follow-up has been associated with differences in smoking/quitting beliefs, including the intention to quit, self-efficacy, and interest in receiving risk information [38]. Additionally, subgroups of pregnant women who have consistently had low retention 
rates include single, less educated, alcohol and drug users, non-working, and not enrolled in WIC programs [37].

Year 1 findings suggest that CTTP is an effective program for pregnant women who stay with the program [81.6\% report not smoking at a median of 6.2 months after achieving 8-week PA (Fig. 2)]. Despite this, retention represents a programmatic limitation of the model in its current form. Future studies will explore this challenge through theorydriven consideration of implementation science and individual outcomes.

\section{Implementation Science Outcomes}

Increasingly, there has been a call for the inclusion of implementation strategies to inform the uptake and impact of evidence-based smoking cessation programs [19, 39]. However, in the synthesized evidence, individual-level (vs. populationlevel) smoking cessation interventions for pregnant women have tended to differ substantially relevant to main intervention strategies, intensity, duration, and in the people involved in their implementation $[5,18]$. The format employed during the first year of the CTTP incorporated multiple strategies. However, while program completion was initially defined as having completed eight visits with prolonged abstinence (8-week PA), the number of sessions employed in the CTTP model varied in succeeding years. Therefore, in subsequent analyses of the CTTP cohort, we plan to evaluate the impact of a varied number of sessions on individual smoking cessation outcomes. In addition, we plan to characterize and evaluate the outcomes among a subsample who repeated the program. These analyses can inform the real-world effectiveness of these program adaptations [22, 40].

As a part of program improvement, there is a need to refine referral processes to ensure more effective contact procedures. Subsequent analyses of the CTTP cohort will evaluate how participants heard about the program and the impact of distance on retention and cessation outcomes, i.e., distance between referring healthcare location and home. There is also an opportunity to explore how the dropout rate changed over time and evaluate the impact of various program iterations on retention.

There is growing evidence that mobile phone text messaging and app-based interventions result in improved quit rates compared with minimal smoking cessation support [41]. However, in a 2019 systematic review of studies on the use of mobile phones and smoking cessation, only one of the 26 studies specifically targeted pregnant women and mothers [41]. Therefore, in order to improve program retention, a text messaging sub-study was conceptualized. Subsequent analyses will report on the effect of introducing mHealth and evaluate this by language (English vs. Spanish). While further research is indicated, these platforms may provide a mechanism for enhancing the reach and engagement with
CTTP participants between face-to-face sessions or as an integrated strategy in developing revised multicomponent models.

Finally, qualitative evaluation methods can further explore CTTP participant's perceptions of what worked and that which did not support their cessation efforts. These mixed methods would also explore the impact of COVID-19 pandemic conditions on participants' ongoing (2020-2021) smoking cessation efforts and their implications for future smoking cessation programs [42, 43].

\section{Individual Outcomes}

In the CTTP cohort, we will be able to report on birth and preterm birth outcomes, including admission to NICU. Our group has previously shown at the county level that for every 35 women who quit smoking, one preterm birth is prevented [44]. At the same time, the estimated cost of running the CTTP was approximately USD 100 versus one approximately 1 million USD for the care of a preterm birth representing substantial savings in healthcare costs [44]. With the CTTP cohort data, we can further quantify the program's direct impact on the county's preterm birth rate. For example, we can explore the association between successful program completion, avoidance of relapse, and prevention of low-birth-weight. In addition, we will have the opportunity to explore the relationship between the range of individual and programmatic variables with these birth outcomes.

In addition, we will determine the effects of the mother's environment on cessation by evaluating the impact of partner and household member's smoking status on maternal smoking cessation during pregnancy and postpartum. Currently, the CTTP model is focused primarily on the mother; however, these findings can inform future program adaptations that would expand the unit of intervention to the household level.

\section{Limitations}

An immediate limitation is that our CTTP evaluation is a retrospective cohort study that is uncontrolled and thus should not be used to estimate efficacy. We note, however, that our objective is to examine the real-world effectiveness of the multicomponent CTTP intervention where components have known efficacy. In addition, the high loss to follow-up rate of the CTTP intervention is a limitation of the current intervention model in terms of scalability since there is the systematic bias of those who dropped out being different. Our objective in future analyses of this cohort is to study this loss to follow-up rate as an implementation science outcome. Furthermore, it is important to note that our follow-up outcomes (up to 9 months after program completion/dropout) represent self-reported point-prevalence abstinence and we 
do not have measures of prolonged continuous abstinence. Finally, while the first-year findings are a good indicator of initial study findings, we acknowledge that these may change with analysis of the entire cohort.

\section{Conclusions}

Our findings support the effectiveness of the CTTP's multicomponent maternal smoking cessation model for achieving 8-week PA but had a high dropout rate. Further evaluation of the entire cohort and analysis of the program implementation and individual participant outcomes can be used to optimize the program.

Author Contributions Conceptualization, PNS and TO; Methodology, PNS; Software, TO and SW; Validation, SW; Formal Analysis, PNS; Writing - Original Draft Preparation, TO; Writing_Review \& Editing, ABP, JY and JCB; Supervision, PNS; Project Administration, PNS; Funding Acquisition, PNS.

Funding Data collection was funded by First 5 CA.gov (San Bernardino). Analysis and reporting by P.S. was funded by Grant HHSN $267200700021 \mathrm{C}$ from NICHD/Department of Health and Human Services (National Children's Study Award to University of California, Irvine, and sub-award to Loma Linda University and California State University San Bernardino).

Data Availability Outcomes from the first-year were presented in a preprint report and can be found here https://www.preprints.org/manus cript/202001.0150/v1. The data that support the findings of this study are available from the corresponding author, $[\mathrm{ABP}]$, upon reasonable request.

\section{Declarations}

Conflict of interest The authors declare no conflict of interest.

Ethical Approval The study was conducted according to the guidelines of the Declaration of Helsinki, and approved by the Institutional Review Board of Loma Linda University (IRB \# 5190418; October $25,2019)$.

Informed Consent Informed consent was obtained from all subjects involved in the study.

\section{References}

1. Lange, S., Probst, C., Rehm, J., \& Popova, S. (2018). National, regional, and global prevalence of smoking during pregnancy in the general population: A systematic review and meta-analysis. The Lancet Global Health., 6(7), e769-e776.

2. U.S. Department of Health and Human Services. (2014). The health consequences of smoking: 50 years of progress. A Report of the Surgeon General. Atlanta: U.S: Department of Health and Human Services, Centers for Disease Control and Prevention,
National Center for Chronic Disease Prevention and Health Promotion, Office on Smoking and Health.

3. Gould, G. S., Havard, A., \& Lim, L. L. (2020). Exposure to tobacco, environmental tobacco smoke and nicotine in pregnancy: A pragmatic overview of reviews of maternal and child outcomes, effectiveness of interventions and barriers and facilitators to quitting. International Journal of Environmental Research Public Health, 17(6), 2034. https://doi.org/10.3390/ ijerph17062034

4. Nighbor, T. D., Doogan, N. J., Roberts, M. E., et al. (2018). Smoking prevalence and trends among a US national sample of women of reproductive age in rural versus urban settings. PLOS ONE, 13(11), e0207818. https://doi.org/10.1371/journal.pone.0207818

5. Chamberlain, C., O'Mara-Eves, A., Porter, J., et al. (2017). Psychosocial interventions for supporting women to stop smoking in pregnancy. Cochrane Database Systematic Reviews, 2, CD001055. https://doi.org/10.1002/14651858.CD001055.pub5

6. Wagijo, M. A., Sheikh, A., Duijts, L., \& Been, J. V. (2017). Reducing tobacco smoking and smoke exposure to prevent preterm birth and its complications. Paediatric Respiratory Review, 22, 3-10. https://doi.org/10.1016/j.prrv.2015.09.002

7. Cnattingius, S., Granath, F., Petersson, G., \& Harlow, B. L. (1999). The influence of gestational age and smoking habits on the risk of subsequent preterm deliveries. J New England Journal of Medicine, 341(13), 943-948.

8. Cooper, S., Orton, S., Leonardi-Bee, J., et al. (2017). Smoking and quit attempts during pregnancy and postpartum: a longitudinal UK cohort. Journal of BMJ Open, 7(11), e018746.

9. Solomon, L. J., \& Quinn, V. P. (2004). Spontaneous quitting: Self-initiated smoking cessation in early pregnancy. Nicotine \& Tobacco Research., 6(Supp12), S203-S216. https://doi.org/10. 1080/14622200410001669132

10. Tong, V. T., Dietz, P. M., Morrow, B., et al. (2013). Trends in smoking before, during, and after pregnancy-Pregnancy Risk Assessment Monitoring System, United States, 40 sites, 20002010. Morbidity and Mortality Weekly Report., 62(6), 1-19.

11. Diamanti, A., Papadakis, S., Schoretsaniti, S., et al. (2019). Smoking cessation in pregnancy: An update for maternity care practitioners. Tobacco Induced Diseases, 17, 57. https://doi.org/10. 18332/tid/109906

12. Bauld, L., Graham, H., Sinclair, L., et al. (2017). Barriers to and facilitators of smoking cessation in pregnancy and following childbirth: Literature review and qualitative study. Health Technology Assessment, 21(36), 1-158. https://doi.org/10.3310/hta21 360

13. Boucher, J., \& Konkle, A. (2016). Understanding inequalities of maternal smoking-bridging the gap with adapted intervention strategies. International Journal of Environmental Research Public Health, 13(3), 282.

14. Riaz, M., Lewis, S., Naughton, F., \& Ussher, M. (2018). Predictors of smoking cessation during pregnancy: A systematic review and meta-analysis. Addiction, 113(4), 610-622. https://doi.org/10. 1111/add.14135

15. Scheuermann, T. S., Richter, K. P., Jacobson, L. T., \& Shireman, T. I. (2017). Medicaid coverage of smoking cessation counseling and medication is underutilized for pregnant women. Nicotine \& Tobacco Research, 19(5), 656-659. https://doi.org/10.1093/ntr/ ntw263

16. Bar-Zeev, Y., Bonevski, B., Lim, L. L., et al. (2019). Improving health providers smoking cessation care in pregnancy: A systematic review and meta-analysis. Addictive Behaviors, 93, 29-38. https://doi.org/10.1016/j.addbeh.2019.01.002

17. Campbell, K. A., Fergie, L., Coleman-Haynes, T., et al. (2018). Improving behavioral support for smoking cessation in pregnancy: What are the barriers to stopping and which behavior change techniques can influence these? Application of theoretical domains 
framework. International Journal of Environmental Research Public Health, 15(2), 359. https://doi.org/10.3390/ijerph15020359

18. US Preventive Services Task Force. (2021). Interventions for tobacco smoking cessation in adults, including pregnant persons: US preventive services task force recommendation statement. JAMA, 325(3), 265-279. https://doi.org/10.1001/jama.2020.25019

19. Mettert, K., Lewis, C., Dorsey, C., Halko, H., \& Weiner, B. (2020). Measuring implementation outcomes: An updated systematic review of measures' psychometric properties. Implementation Research and Practice, 1, 2633489520936644. https://doi.org/10. $1177 / 2633489520936644$

20. Eccles, M. P., \& Mittman, B. S. (2006). Welcome to implementation science. Implementation Science, 1(1), 1. https://doi.org/10. 1186/1748-5908-1-1

21. Nilsen, P., \& Birken, S. A. (2020). Handbook on implementation science. Chwltenham: Edward Elgar Publishing.

22. Brownson, R. C., Colditz, G. A., \& Proctor, E. K. (2012). Dissemination and implementation research in health: Translating science to practice. Oxford University Press.

23. American College of Obstetricians and Gynecologists. (2020). Tobacco and nicotine cessation during pregnancy. ACOG Committee Opinion Number 807. Obstetrics \& Gynecology, 135(5), e221-e229.

24. Gaalema, D. E., Higgins, S. T., Bradstreet, M. P., Heil, S. H., \& Bernstein, I. M. (2011). Using NicAlert strips to verify smoking status among pregnant cigarette smokers. Drug and Alcohol Dependence, 119(1-2), 130-133. https://doi.org/10.1016/j.druga lcdep.2011.05.014

25. Cox, J. L., Holden, J. M., \& Sagovsky, R. (1987). Detection of postnatal depression. Development of the 10-item Edinburgh Postnatal Depression Scale. British Journal of Psychiatry., 150, 782-786. https://doi.org/10.1192/bjp.150.6.782

26. Wisner, K. L., Parry, B. L., \& Piontek, C. M. (2002). Clinical practice. Postpartum depression. New England Journal of Medicine, 347(3), 194-199. https://doi.org/10.1056/NEJMcp011542

27. The American Congress of Obstetricians and Gynecologists. (2017). Committee Opinion No. 721: Smoking cessation during pregnancy. Obstetrician and Gynecologist, 130(4), e200-e204. https://doi.org/10.1097/aog.0000000000002353

28. SAS Institute Inc. (2011). SAS®9.3. Cary: SAS Institute Inc.

29. California Department of Public Health. (2018). Maternal and Infant Health Assessment (MIHA) Survey Data Snapshot, San Bernardino County, 2013-2015.

30. California Department of Public Health. (2015). Maternal and Infant Health Assessment (MIHA); Statewide Data Snapshots, 2013-2015.

31. California Department of Public Health. (2019). California American Indian/Alaska Native Maternal and Infant Health Status Report.

32. Drake, P., Driscoll, A. K., \& Mathews, T. J. (2018). Cigarette smoking during pregnancy: United States, 2016. NCHS Data Brief, no 305. U.S.: National Center for Health Statistics

33. Frazer, K., Fitzpatrick, P., Brosnan, M., et al. (2020). Smoking prevalence and secondhand smoke exposure during pregnancy and postpartum-establishing risks to health and human rights before developing a tailored programme for smoking cessation. International Journal of Environmental Research Public Health, 17(6), 1838. https://doi.org/10.3390/ijerph17061838
34. Alvidrez, J., Castille, D., Laude-Sharp, M., Rosario, A., \& Tabor, D. (2019). The National Institute on Minority Health and Health Disparities research framework. American Journal of Public Health, 109(S1), S16-S20. https://doi.org/10.2105/ajph.2018. 304883

35. Windsor, R. (2010). Behavioral treatment methods for pregnant smokers: the evidence base for prenatal care programs and professional practice. In A. Handler, J. Kennelly, \& N. Peacock (Eds.), School of Public Health. University of Illinois, Springer.

36. Windsor, R., Woodby, L., Miller, T., \& Hardin, M. (2011). Effectiveness of Smoking Cessation and Reduction in Pregnancy Treatment (SCRIPT) methods in Medicaid-supported prenatal care: Trial III. Health Education \& Behavior, 38(4), 412-422. https:// doi.org/10.1177/1090198110382503

37. El-Khorazaty, M. N., Johnson, A. A., Kiely, M., et al. (2007). Recruitment and retention of low-income minority women in a behavioral intervention to reduce smoking, depression, and intimate partner violence during pregnancy. BMC Public Health, 7, 233. https://doi.org/10.1186/1471-2458-7-233

38. Emery, J. L., Sutton, S., \& Naughton, F. (2017). Cognitive and behavioral predictors of quit attempts and biochemically-validated abstinence during pregnancy. Nicotine \& Tobacco Research., 19(5), 547-554. https://doi.org/10.1093/ntr/ntw242

39. Selove, R., Neil-Sztramko, S., Leng, J., et al. (2020). Use of implementation science in tobacco control intervention studies in the USA between 2000 and 2020: A scoping review protocol. British Medical Journal Open, 10(11), e038617. https://doi.org/10.1136/ bmjopen-2020-038617

40. Koskinen, K., Hytönen, M., \& Räsänen, P. (2020). The use of realworld effectiveness measurements in clinical settings at Helsinki University Hospital: A systematic literature review. Journal of Comparative Effectiveness Research, 9(5), 321-326. https://doi. org/10.2217/cer-2019-0159

41. Whittaker, R., McRobbie, H., Bullen, C., Rodgers, A., Gu, Y., \& Dobson, R. (2019). Mobile phone text messaging and app-based interventions for smoking cessation. The Cochrane Systematic Review, 10, CD006611. https://doi.org/10.1002/14651858.CD006 611.pub5

42. He, Y., Sun, J., Ding, X., \& Wang, Q. (2021). Mechanisms in which smoking increases the risk of COVID-19 infection: A narrative review. Iran Journal Public Health, 50(3), 431-437. https:// doi.org/10.18502/ijph.v50i3.5582

43. van Zyl-Smit, R. N., Richards, G., \& Leone, F. T. (2020). Tobacco smoking and COVID-19 infection. Lancet Respiratory Medicine., 8(7), 664-665. https://doi.org/10.1016/s2213-2600(20)30239-3

44. Batech, M., Tonstad, S., Job, J. S., et al. (2013). Estimating the impact of smoking cessation during pregnancy: The San Bernardino County experience. Journal of Community Health, 38(5), 838-846. https://doi.org/10.1007/s10900-013-9687-8

Publisher's Note Springer Nature remains neutral with regard to jurisdictional claims in published maps and institutional affiliations. 